\title{
Star Formation Histories from Pan-Chromatic Infrared Continuum Surveys
}

\author{
Sergio Molinari ${ }^{1}$ \\ ${ }^{1}$ INAF - Istituto Fisica Spazio Interplanetario, Via Fosso del Cavaliere 100, 00133 Rome, Italy \\ email: molinari@ifsi-roma.inaf.it
}

\begin{abstract}
One of the currently most disputed issues in Star Formation is the timeline of the whole process. Is it a "slow" process of cloud assembly which, mediated by magnetic fields, evolve toward turbulence-supported clumps which are eventually super-critical to collapse, e.g. McKee \& Tan (2003)? Or do clumps originate in already super-critical state in the post-shock regions of large-scale Galactic converging flows, e.g. Hartmann et al. (2001) with a rapid collapse in a crossing time or so (Elmegreen 2000)?

A pan-chromatic $1 \mu \mathrm{m}-1 \mathrm{~mm}$ continuum view of cluster forming regions in their early stages offers access to the most massive members longward of $5-10 \mu \mathrm{m}$, as well as the low-mass members which instead dominate the emission in the near-IR, offering an interesting potential in stimulating advances in theoretical modelling of clustered star formation, its history and rate.
\end{abstract}

Keywords. stars: formation

Molinari et al. (2008) used mid-IR to submm images to reconstruct the Spectral Energy Distribution (SED) and luminosity for the dominant YSOs in 42 high-mass star forming regions from Molinari et al. (1996), and used extensive radiative transfer modelling to disentangle massive YSOs in their hot-core or UCHII stage, from massive YSOs still in their active pre-ZAMS accretion phase. The modelled timescales for the formation are in the range 1 to a few $10^{5}$ years depending on the mass.

In a recent analysis of near-IR images toward 26 high-mass star forming regions (also from Molinari et al. (1996)) Faustini et al. (2009) found a cluster of low mass objects associated with the massive YSOs in $80 \%$ of the cases. An extensive grid of Monte Carlo simulations was used to try and deduce fundamental cluster parameters, including indications on their ages. In less than a dozen clusters where the analysis delivered significant results, the median age of the cluster members was found between 1 and a few $10^{6}$ years, with an indication for a spread of ages of similar magnitude within each cluster.

Taken at face value, the ages estimated for the low and high-mass members of young embedded clusters seem inconsistent with a single burst of star formation and suggest that low-mass members are the first to start collapse, and that the entire cluster formation process encompasses several dynamical timescales. Therefore this is difficult to reconcile with fast formation scenarios.

\section{References}

Elmegreen, B. G. 2000, ApJ 530, 277

Faustini, F., Molinari, S., Brand, J., \& Testi, L. 2009, A\&A 503, 801

Hartmann, L., Ballesteros-Paredes, J., \& Bergin, E. A. 2001, ApJ 562, 852

McKee, C. F. \& Tan, J. C. 2003, ApJ 585, 850

Molinari, S., Brand, J., Cesaroni, R., \& Palla, F. 1996, A\&A 308, 573

Molinari, S., Pezzuto, S., Cesaroni, R., Brand, J., Faustini, F., \& Testi, L. 2008, A\&A 481, 345 